KYUNGPOOK Math. J. 53(2013), 479-495

http://dx.doi.org/10.5666/KMJ.2013.53.3.479

\title{
Weighted Carlson Mean of Positive Definite Matrices
}

\author{
Hosoo Lee \\ Department of Mathematics, Louisiana State University, Baton Rouge, LA70803, \\ $U S A$ \\ e-mail : hosoolee@lsu.edu
}

Abstract. Taking the weighted geometric mean [11] on the cone of positive definite matrix, we propose an iterative mean algorithm involving weighted arithmetic and geometric means of $n$-positive definite matrices which is a weighted version of Carlson mean presented by Lee and Lim [13]. We show that each sequence of the weigthed Carlson iterative mean algorithm has a common limit and the common limit of satisfies weighted multidimensional versions of all properties like permutation symmetry, concavity, monotonicity, homogeneity, congruence invariancy, duality, mean inequalities.

\section{Introduction}

For positive real numbers $a$ and $b$, the sequences $\left\{a_{n}\right\}$ and $\left\{b_{n}\right\}$ defined by

$$
a_{0}=a, \quad b_{0}=b, \quad a_{n+1}=\frac{1}{2}\left(a_{n}+b_{n}\right), \quad b_{n+1}=\sqrt{a_{n+1} b_{n}}
$$

converge to a common limit. This is called Borchardt's algorithm [5]. In a generalization of Borchardt's algorithm with a suitable incomplete elliptic integral representation and with "permutation symmetry," Carlson [6] has found a 3-dimensional iterative mean algorithm involving arithmetic and geometric means of positive reals. For positive reals $a_{0}=a, b_{0}=b$ and $c_{0}=c$, the three sequences defined by

$$
\begin{aligned}
a_{n+1} & =\left(\frac{a_{n}+b_{n}}{2} \frac{a_{n}+c_{n}}{2}\right)^{\frac{1}{2}}, \\
b_{n+1} & =\left(\frac{b_{n}+c_{n}}{2} \frac{a_{n}+b_{n}}{2}\right)^{\frac{1}{2}}, \\
c_{n+1} & =\left(\frac{a_{n}+c_{n}}{2} \frac{b_{n}+c_{n}}{2}\right)^{\frac{1}{2}}
\end{aligned}
$$

* Corresponding Author.

Received June 27,2013; accepted August 01, 2013.

2010 Mathematics Subject Classification: 65F30, 15A48, 47A64, 20 B35.

Key words and phrases: Positive definite matrix, matrix geometric mean, iterative mean algorithm, weighted symmetrization procedure. 
approach to a common limit. This satisfies the permutation symmetry and includes Borchardt's algorithm (when $b=c$ ) and the common limit has a symmetric integral representation. A key observation in the Carlson algorithm is that it is a composition of two symmetrization procedures: $\left(a_{n+1}, b_{n+1}, c_{n+1}\right)=\delta\left(a_{n}, b_{n}, c_{n}\right)$ where $\delta=\gamma \circ \beta$ and

$$
\beta(a, b, c)=\frac{1}{2}(b+c, a+c, a+b), \quad \gamma(a, b, c)=(\sqrt{b c}, \sqrt{a c}, \sqrt{a b}) .
$$

The geometric mean of two positive definite matrices $A$ and $B$ is defined by $A \# B=A^{1 / 2}\left(A^{-1 / 2} B A^{-1 / 2}\right)^{1 / 2} A^{1 / 2}$ and is regarded as a unique positive definite solution of the Riccati equation $X A^{-1} X=B$ [12]. In the Riemannian manifold of positive definite matrices equipped with the Riemannian metric (it coincides with the Hessian metric of the logarithmic barrier functional) $d s=\left\|A^{-1 / 2} d A A^{-1 / 2}\right\|_{2}=$ $\left(\operatorname{tr}\left(A^{-1} d A\right)^{2}\right)^{1 / 2}([10,2,3,12]), A \# B$ is the unique metric midpoint of $A$ and $B$ for the Riemannian metric distance satisfying the symmetry $A \# B=B \# A$.

q It has been a long-standing problem to extend the two-variable geometric mean of positive definite matrices to $n$-variables, $n \geq 3$, and a variety of attempts may be found in the literature. Two recent approaches have been given by Ando-Li-Mathias [1] and Bini-Meini-Poloni [4] via "symmetrization methods" and induction. From these successful extensions of geometric means of $n$-positive definite matrices, Lee and Lim [13] have generalized Carlson's algorithm from the case that variables vary over 3-dimensinal positive real numbers to the case that variables vary over multivariable positive definite matrices preserving permutation symmetry.

In [11], the authors have constucted a weighted geometric mean $\mathfrak{G}_{n}\left(\omega ; A_{1}, \ldots, A_{n}\right)$ where $\omega$ varies over $n$-dimentional positive probability vectors via weighted version of Bini-Meini-Poloni symmetrization procedure and induction, satisfying the weighted version of ten properties of ALM geometric mean.

The main purpose of this paper is to propose weighted Carlson means based on a weighted version of the generalized permutation symmetrization procedure in [13]. For a positive probability vector $\omega=\left(w_{1}, \ldots, w_{n}\right)$ and an $n$-tuple of positive definite matrices $A=\left(A_{1}, \ldots, A_{n}\right)$, our symmetrization method is given by

$$
\begin{aligned}
\left(A_{1}^{(0)}, \ldots, A_{n}^{(0)}\right) & =\left(A_{1}, \ldots, A_{n}\right) \\
\left(A_{1}^{(r+1)}, \ldots, A_{n}^{(r+1)}\right) & =\delta_{\omega}\left(A_{1}^{(r)}, \ldots, A_{n}^{(r)}\right),
\end{aligned}
$$

where $\delta_{\omega}=\gamma_{\omega} \circ \beta_{\omega}, \beta_{\omega}$ the weighted Ando-Li-Mathias symmetrization procedure of arithmetic mean and $\gamma_{\omega}$ is the weighted Bini-Meini-Poloni symmetrization procedure of geometric mean. We show that the sequences $\left\{A_{i}^{(r)}\right\}_{r=0}^{\infty}, i=1, \ldots, n$, converge to a common limit, yielding a weighted Carlson mean $\mathfrak{C}_{n}(\omega ; A)$. In Section 4 , we present all properties of the weighted Carlson mean of positive definite matrices. 


\section{The Convex Cone of Positive Definite Matrices}

Let $\mathrm{M}(m)$ be the space of $m \times m$ complex matrices equipped with the operator norm $\|\cdot\|, \mathrm{H}(m)$ the space of $m \times m$ complex Hermitian matrices, and $\Omega=\Omega(m)$ the convex cone of positive definite Hermitian matrices. The general linear group $\operatorname{GL}(m, C)$ acts on $\Omega(m)$ transitively via congruence transformations $\Gamma_{M}(X)=M X M^{*}$. For $X, Y \in \mathrm{H}(m)$, we write that $X \leq Y$ if $Y-X$ is positive semidefinite, and $X<Y$ if $Y-X$ is positive definite (positive semidefinite and invertible). Each positive semidefinite matrix $A$ has a unique positive semidefinite square root, denoted by $A^{1 / 2}$. For $A \in \mathrm{H}(m), \lambda_{j}(A)$ are the eigenvalues of $A$ in non-increasing order: $\lambda_{1}(A) \geq \lambda_{2}(A) \geq \cdots \geq \lambda_{m}(A)$. We note that the operator norm of a Hermitian matrix $A$ coincides with its spectral norm $\|A\|=\max \left\{\left|\lambda_{j}(A)\right|: 1 \leq j \leq m\right\}$. The Thompson metric given by

$$
d(A, B)=\max \{\log M(B / A), \log M(A / B)\},
$$

where $M(B / A)=\inf \{\alpha>0: B \leq \alpha A\}=\lambda_{1}\left(A^{-1 / 2} B A^{-1 / 2}\right)$ is a complete metric on the open convex cone $\Omega=\Omega(m)$. For $0<A \leq B$, we denote $[A, B]=\{X>0$ : $A \leq X \leq B\}$.

Lemma 2.1. Let $0<A \leq B$. If $X, Y \in[A, B]$ then $d(X, Y) \leq d(A, B)$.

Lemma 2.2. The Thompson metric on $\Omega(m)$ satisfies

$$
d\left(\sum_{i=1}^{n} A_{i}, \sum_{i=1}^{n} B_{i}\right) \leq \max \left\{d\left(A_{i}, B_{i}\right)\right\}_{i=1}^{n}
$$

for any $A_{i}, B_{i} \in \Omega(m), 1 \leq i \leq n$.

The following additive contraction theorem will play a key role for our purpose.

Proposition 2.3.[[14]] Let $A$ be a $l \times l$ positive semidefinite matrix. Then

$$
d(A+X, A+Y) \leq \frac{\alpha}{\alpha+\beta} d(X, Y), \quad X, Y \in \Omega(m)
$$

where $\alpha=\max \left\{\lambda_{1}(X), \lambda_{1}(Y)\right\}$ and $\beta=\lambda_{l}(A)$.

\section{Weighted Geometric Means of Positive Definite Matrices}

The curve $t \mapsto A \#_{t} B:=A^{1 / 2}\left(A^{-1 / 2} B A^{-1 / 2}\right)^{t} A^{1 / 2}$ is a minimal geodesic line between $A$ and $B$ and its geodesic middle $A \# B:=A \#_{1 / 2} B$ is known as the geometric mean of $A$ and $B$. 
Theorem 3.1.[[2]] For $A, B, C \in \Omega$ and $M \in \mathrm{GL}(m, C)$,

(1) $d(A, B)=d\left(A^{-1}, B^{-1}\right)=d\left(M A M^{*}, M B M^{*}\right)$;

(2) $d(A \# B, A)=d(A \# B, B)=\frac{1}{2} d(A, B)$;

(3) for all $s, t \in[0,1], d\left(A \#_{t} B, A \#_{s} B\right)=|s-t| d(A, B)$ and

$$
d\left(A \#_{t} B, C \#_{t} D\right) \leq(1-t) d(A, C)+t d(B, D) .
$$

The non-positive curvature property (3.1) for the Thompson metric is appeared in [8] even for positive definite operators on a Hilbert space.

The following properties for the weighted geometric mean $A \#_{t} B$ are well-known.

Lemma 3.2. Let $A, B, C, D \in \Omega$ and let $t \in[0,1]$. Then

(i) $A \#_{t} B=A^{1-t} B^{t}$ if $A B=B A$;

(ii) $(a A) \#_{t}(b B)=a^{1-t} b^{t}\left(A \#_{t} B\right)$ for $a, b>0$;

(iii) (Löwner-Heinz inequality) $A \#_{t} B \leq C \#_{t} D$ if $A \leq C$ and $B \leq D$;

(iv) $M\left(A \#_{t} B\right) M^{*}=\left(M A M^{*}\right) \#_{t}\left(M B M^{*}\right)$ for non-singular $M$;

(v) $A \#_{t} B=B \#_{1-t} A,\left(A \#_{t} B\right)^{-1}=A^{-1} \#_{t} B^{-1}$;

(vi) $(\lambda A+(1-\lambda) B) \#_{t}(\lambda C+(1-\lambda) D) \geq \lambda\left(A \#_{t} C\right)+(1-\lambda)\left(B \#_{t} D\right)$ for $\lambda \in[0,1]$;

(vii) $\operatorname{det}\left(A \#_{t} B\right)=\operatorname{det}(A)^{1-t} \operatorname{det}(B)^{t}$;

(viii) $\left((1-t) A^{-1}+t B^{-1}\right)^{-1} \leq A \#_{t} B \leq(1-t) A+t B$.

$A \#{ }_{t} B$ as a two-variable weighted mean, denoted by $\mathfrak{G}_{2}(1-t, t ; A, B)$, the authors of [11] have constucted for each $n>2$ a weighted geometric mean $\mathfrak{G}_{n}\left(\omega ; A_{1}\right.$, $\left.\ldots, A_{n}\right)$, where $\omega=\left(w_{1}, \ldots, w_{n}\right)$ varies over $n$-dimentional positive probability vectors via weighted version of Bini-Meini-Poloni symmetrization procedure [4]. Let $\left(w_{1}, w_{2}, w_{3}\right)$ be a positive probability vector and let $A_{1}, A_{2}, A_{3}$ be positive definite matrices. Starting with $\left(A_{1}^{(0)}, A_{2}^{(0)}, A_{3}^{(0)}\right)=\left(A_{1}, A_{2}, A_{3}\right)$ define

$$
\begin{aligned}
& \left(A_{1}^{(1)}, A_{2}^{(1)}, A_{3}^{(1)}\right)=\left(A_{1} \#_{1-w_{1}}\left(A_{2} \#_{\frac{w_{3}}{1-w_{1}}} A_{3}\right),\right. \\
& A_{2} \#_{1-w_{2}}\left(A_{1} \# \frac{w_{3}}{1-w_{2}} A_{3}\right) \text {, } \\
& \left.A_{3} \#_{1-w_{3}}\left(A_{1} \# \frac{w_{2}}{1-w_{3}} A_{2}\right)\right) \text {, } \\
& \left(A_{1}^{(r)}, A_{2}^{(r)}, A_{3}^{(r)}\right)=\left(A_{1}^{(r-1)} \#_{1-w_{1}}\left(A_{2}^{(r-1)} \# \frac{w_{3}}{1-w_{1}} A_{3}^{(r-1)}\right),\right. \\
& A_{2}^{(r-1)} \#_{1-w_{2}}\left(A_{1}^{(r-1)} \# \frac{w_{3}}{1-w_{2}} A_{3}^{(r-1)}\right) \text {, } \\
& \left.A_{3}^{(r-1)} \#_{1-w_{3}}\left(A_{1}^{(r-1)} \# \frac{w_{2}}{1-w_{3}} A_{2}^{(r-1)}\right)\right) \text {. }
\end{aligned}
$$


It is shown that the sequences $\left\{A_{i}^{(r)}\right\}_{r=0}^{\infty}, i=1,2,3$, converge to a common limit, yielding geometric means of 3 -positive definite matrices $\mathfrak{G}_{3}\left(w_{1}, w_{2}, w_{3} ; A_{1}, A_{2}, A_{3}\right)$. Inductively, for $n$-dimensional positive probability vector $\omega=\left(w_{1}, w_{2}, \ldots, w_{n}\right)$, the weighted symmetrization procedure of $n$-positive definite matrices is defined by

$$
\gamma_{\omega}(A)=\left(A_{1} \#_{1-w_{1}} \mathfrak{G}_{n-1}\left(\hat{\omega}_{\neq 1} ; A_{k \neq 1}\right), \ldots, A_{n} \#_{1-w_{n}} \mathfrak{G}_{n-1}\left(\hat{\omega}_{\neq n} ; A_{k \neq n}\right)\right)
$$

where $\hat{\omega}_{\neq i}=\frac{1}{1-w_{i}}\left(w_{1}, \ldots, w_{i-1}, w_{i+1}, \ldots, w_{n}\right)$ is $(n-1)$-dimensional positive probability vector and $A_{k \neq i}=\left(A_{1}, \ldots, A_{i-1}, A_{i+1}, \ldots, A_{n}\right)$ of $A=\left(A_{1}, \ldots, A_{n}\right)$. Then each component of the iteration $\gamma_{\omega}^{r}(A)=\left(A_{1}^{(r)}, \ldots, A_{n}^{(r)}\right)$ approaches to a common limit, yielding $n$-dimensional weighted geometric mean $\mathfrak{G}_{n}(\omega ; A)$.

The weighted geometric means of $n$-positive definite matrices satisfy the following properties.

Theorem 3.3. Let $A=\left(A_{1}, A_{2}, \ldots, A_{n}\right), B=\left(B_{1}, B_{2}, \ldots, B_{n}\right) \in \Omega^{n}$ and let $\omega=\left(w_{1}, w_{2}, \ldots, w_{n}\right)$ be a positive probability vector.

(P1) $\mathfrak{G}_{n}\left(\omega ; A_{1}, \ldots A_{n}\right)=A_{1}^{w_{1}} \cdots A_{n}^{w_{n}}$ for commuting $A_{i}$ 's.

(P2) (Joint homogeneity)

$$
\mathfrak{G}_{n}\left(\omega ; a_{1} A_{1}, \ldots, a_{n} A_{n}\right)=a_{1}^{w_{1}} \cdots a_{n}^{w_{n}} \mathfrak{G}_{n}\left(\omega ; A_{1}, \ldots, A_{n}\right) .
$$

(P3) (Permutation invariance) For any permutation $\sigma$,

$$
\mathfrak{G}_{n}\left(\omega_{\sigma} ; A_{\sigma(1)}, \ldots, A_{\sigma(n)}\right)=\mathfrak{G}_{n}\left(\omega ; A_{1}, \ldots, A_{n}\right) .
$$

(P4) (Monotonicity) If $B_{i} \leq A_{i}$ for all $1 \leq i \leq n$, then

$$
\mathfrak{G}_{n}\left(\omega ; B_{1}, \ldots, B_{n}\right) \leq \mathfrak{G}_{n}\left(\omega ; A_{1}, \ldots, A_{n}\right) .
$$

(P5) (Continuity) The map $\mathfrak{G}_{n}(\omega ; \cdot)$ is continuous.

(P6) (Congruence invariance) For any invertible matrix $M$,

$$
\mathfrak{G}_{n}\left(\omega ; M A_{1} M^{*}, \ldots, M A_{n} M^{*}\right)=M \mathfrak{G}_{n}\left(\omega ; A_{1}, \ldots, A_{n}\right) M^{*} .
$$

(P7) (Joint Concavity) For $0 \leq \lambda \leq 1$,

$$
\begin{aligned}
& \mathfrak{G}_{n}\left(\omega ; \lambda A_{1}+(1-\lambda) B_{1}, \ldots, \lambda A_{n}+(1-\lambda) B_{n}\right) \\
& \quad \geq \lambda \mathfrak{G}_{n}\left(\omega ; A_{1}, \ldots, A_{n}\right)+(1-\lambda) \mathfrak{G}_{n}\left(\omega ; B_{1}, \ldots, B_{n}\right) .
\end{aligned}
$$

(P8) (Self-duality) $\mathfrak{G}_{n}\left(\omega ; A_{1}^{-1}, \ldots, A_{n}^{-1}\right)=\mathfrak{G}_{n}\left(\omega ; A_{1}, \ldots, A_{n}\right)^{-1}$.

(P9) (Determinantal identity) $\operatorname{det}\left(\mathfrak{G}_{n}\left(\omega ; A_{1}, \ldots, A_{n}\right)\right)=\prod_{i=1}^{n}\left(\operatorname{det} A_{i}\right)^{\omega_{i}}$. 
(P10) (Arithmetic-geometric-harmonic mean inequality)

$$
\left(\sum_{i=1}^{n} w_{i} A_{i}^{-1}\right)^{-1} \leq \mathfrak{G}_{n}\left(\omega ; A_{1} \ldots, A_{n}\right) \leq \sum_{i=1}^{n} w_{i} A_{i} .
$$

We call a mean of $n$-variables satisfying these properties a weighted geometric mean. We note that for $\omega=(1 / n, \ldots, 1 / n)$ the geometric means obtained by AndoLi-Mathias [1] and by Bini-Meini-Poloni [4] satisfy (P1)-(P10).

Remark 3.4. In [11], the authors obtained a stronger version of (P5) for the Thompson metric;

$(\mathrm{P} 11) d\left(\mathfrak{G}_{n}\left(\omega ; A_{1}, \ldots, A_{n}\right), \mathfrak{G}_{n}\left(\omega ; B_{1}, \ldots, B_{n}\right)\right) \leq \sum_{i=1}^{n} w_{i} d\left(A_{i}, B_{i}\right)$.

Proposition 3.5. Let $\omega=\left(w_{1}, \ldots, w_{n}\right)$ and $\nu=\left(v_{1}, \ldots, v_{n}\right)$ be positive probability vectors. Then

$$
d\left(\mathfrak{G}_{n}\left(\omega ; A_{1}, \ldots, A_{n}\right), \mathfrak{G}_{n}\left(\nu ; B_{1}, \ldots, B_{n}\right)\right) \leq \sum_{i, j=1}^{n} w_{i} v_{j} d\left(A_{i}, B_{j}\right) .
$$

Proof. By (P1) and (P11), we have

$$
\begin{aligned}
d\left(\mathfrak{G}_{n}\left(\omega ; A_{1}, \ldots, A_{n}\right), \mathfrak{G}_{n}\left(\nu ; B_{1}, \ldots, B_{n}\right)\right) \\
\quad=d\left(\mathfrak{G}_{n}\left(\omega ; A_{1}, \ldots, A_{n}\right), \mathfrak{G}_{n}\left(\omega ; \mathfrak{G}_{n}\left(\nu ; B_{1}, \ldots, B_{n}\right), \ldots, \mathfrak{G}_{n}\left(\nu ; B_{1}, \ldots, B_{n}\right)\right)\right. \\
\quad \leq \sum_{i=1}^{n} w_{i} d\left(A_{i}, \mathfrak{G}_{n}\left(\nu ; B_{1}, \ldots, B_{n}\right)\right) \\
\quad=\sum_{i=1}^{n} w_{i} d\left(\mathfrak{G}_{n}\left(\nu ; A_{i}, \ldots, A_{i}\right), \mathfrak{G}_{n}\left(\nu ; B_{1}, \ldots, B_{n}\right)\right) \\
\left.\quad \leq \sum_{i=1}^{n} \sum_{j=1}^{n} w_{i} v_{j} d\left(A_{i}, B_{j}\right)\right) .
\end{aligned}
$$

\section{Higher Order Weighted Carlson's Algorithm}

Let $\Delta_{n}=\left\{\left(w_{1}, \ldots, w_{n}\right) \in(0,1)^{n}: \sum_{i=1}^{n} w_{i}=1\right\}$ be the set of $n(n \geq 2)$ dimensional positive probability vectors. For $\omega=\left(w_{1}, \ldots, w_{n}\right) \in \Delta_{n}$, we denote

$$
\begin{aligned}
\omega_{\neq j} & =\left(w_{1}, \ldots, w_{j-1}, w_{j+1}, \ldots, w_{n}\right) \\
\omega_{\neq i, j} & =\left(w_{1}, \ldots, w_{i-1}, w_{i+1}, \ldots, w_{j-1}, w_{j+1}, \ldots, w_{n}\right) \quad(i<j) \\
\hat{\omega}_{\neq j} & =\frac{1}{1-w_{j}} \omega_{\neq j} \in \Delta_{n-1}, \quad(n \geq 3)
\end{aligned}
$$


and $w_{j k}=\left\{\begin{array}{cc}\frac{w_{k}}{1-w_{j}}, & \text { if } j \neq k \\ 0, & \text { if } j=k .\end{array}\right.$ Then we have $\hat{\omega}_{\neq j}=\left(\omega_{j 1}, \ldots, \omega_{j(j-1)}, \omega_{j(j+1)}, \ldots, \omega_{j n}\right)$. For $A=\left(A_{1}, \ldots, A_{n+1}\right) \in \Omega^{n+1}$ and $\omega=\left(w_{1}, \ldots, w_{n+1}\right) \in \Delta_{n+1}$, we consider the symmetrization procedure of arithmetic and harmonic means

$$
\begin{gathered}
\beta_{\omega}(A)=\left(\sum_{k=1}^{n+1} w_{1 k} A_{k}, \ldots, \sum_{k=1}^{n+1} w_{(n+1) k} A_{k}\right), \\
\beta_{\omega}^{*}(A)=\left(\left(\sum_{k=1}^{n+1} w_{1 k} A_{k}^{-1}\right)^{-1}, \ldots,\left(\sum_{k=1}^{n+1} w_{(n+1) k} A_{k}^{-1}\right)^{-1}\right) .
\end{gathered}
$$

It is not difficult to see that there exist positive definite matrices $X^{*}$ and $Y^{*}$ such that

$$
\lim _{r \rightarrow \infty} \beta_{\omega}^{r}(A)=\left(X^{*}, \ldots, X^{*}\right) \text { and } \lim _{r \rightarrow \infty}\left(\beta_{\omega}^{*}\right)^{r}(A)=\left(Y^{*}, \ldots, Y^{*}\right) .
$$

Indeed, the map $\beta$ has the linear representation

$$
\left[\begin{array}{c}
A_{1} \\
A_{2} \\
\vdots \\
A_{n+1}
\end{array}\right] \mapsto\left[\begin{array}{ccccc}
0 & w_{12} & w_{13} & \cdots & w_{1(n+1)} \\
w_{21} & 0 & w_{23} & \cdots & w_{2(n+1)} \\
\vdots & \vdots & \vdots & \ddots & \vdots \\
w_{(n+1) 1} & w_{(n+1) 2} & w_{(n+1) 3} & \cdots & 0
\end{array}\right]\left[\begin{array}{c}
A_{1} \\
A_{2} \\
\vdots \\
A_{n+1}
\end{array}\right]
$$

where $w_{i j}=\frac{w_{j}}{1-w_{i}}$ and

$$
\left[\begin{array}{ccccc}
0 & w_{12} & w_{13} & \cdots & w_{1(n+1)} \\
w_{21} & 0 & w_{23} & \cdots & w_{2(n+1)} \\
\vdots & \vdots & \vdots & \ddots & \vdots \\
w_{(n+1) 1} & w_{(n+1) 2} & w_{(n+1) 3} & \cdots & 0
\end{array}\right]^{r} \rightarrow\left[\begin{array}{ccccc}
z_{1} & z_{2} & z_{3} & \cdots & z_{n+1} \\
z_{1} & z_{2} & z_{3} & \cdots & z_{n+1} \\
\vdots & \vdots & \vdots & \vdots & \vdots \\
z_{1} & z_{2} & z_{3} & \cdots & z_{n+1}
\end{array}\right] .
$$

A stochastic matrix is said to be regular if some power has all positive entries. We note that the obtained $(n+1) \times(n+1)$ matrix is a regular stochastic matrix with an eigenvector $z=\left(z_{1}, z_{2}, \ldots, z_{n+1}\right)^{T}$. It is well-known (cf. Chapter 8, [9]) that every regular stochastic matrix $M$ has a unique probability vector $z$ with all positive components such that $M z=z$, and the sequence $\left\{M^{k}\right\}$ converges to a matrix $S$ whose columns are the fixed column vector $z$.

The case $\beta_{\omega}^{*}$ follows from $\beta_{\omega}\left(A^{-1}\right)^{-1}=\beta_{\omega}^{*}(A)$.

Define a self-map on $\Omega^{n+1}$;

$$
\gamma_{\omega}(A)=\left(A_{1} \#_{1-w_{1}} \mathfrak{G}_{n}\left(\hat{\omega}_{\neq 1} ; A_{k \neq 1}\right), \ldots, A_{n+1} \#_{1-w_{n+1}} \mathfrak{G}_{n}\left(A_{k \neq n+1}\right)\right)
$$

where $A=\left(A_{1}, \ldots, A_{n+1}\right) \in \Omega^{n+1}$ and

$$
A_{k \neq i}:=\left(A_{1}, \ldots, A_{i-1}, A_{i+1}, \ldots, A_{n+1}\right) \in \Omega^{n} .
$$


We consider the compositions $\delta_{\omega}$ and $\mu_{\omega}$ on $\Omega^{n+1}$ defined as

$$
\delta_{\omega}:=\gamma_{\omega} \circ \beta_{\omega} \quad \text { and } \quad \mu_{\omega}:=\gamma_{\omega} \circ \beta_{\omega}^{*} .
$$

Theorem 4.1. For each $A \in \Omega^{n+1}$, the sequences $\left\{\delta_{\omega}^{r}\right\}_{r \in N}$ and $\left\{\mu_{\omega}^{r}\right\}_{r \in N}$ are power convergent, i.e., there exist $X^{*}, X_{*} \in \Omega$ such that

$$
\begin{aligned}
& \lim _{r \rightarrow \infty} \delta_{\omega}^{r}(A)=\left(X^{*}, X^{*}, \ldots, X^{*}\right), \\
& \lim _{r \rightarrow \infty} \mu_{\omega}^{r}(A)=\left(X_{*}, X_{*}, \ldots, X_{*}\right) .
\end{aligned}
$$

Proof. Let $A \in \Omega^{n+1}$. Setting $\gamma=\gamma_{\omega}, \delta=\delta_{\omega}$ and

$$
\begin{gathered}
\delta^{r}(A)=\left(A_{1}^{(r)}, A_{2}^{(r)}, \ldots, A_{n+1}^{(r)}\right), \\
\left(\beta \circ \delta^{r}\right)(A)=\left(X_{1}^{(r)}, X_{2}^{(r)}, \ldots, X_{n+1}^{(r)}\right),
\end{gathered}
$$

we have $A_{i}^{(0)}=A_{i}, X_{i}^{(0)}=\sum_{k=1}^{n+1} w_{i k} A_{k}$ and

$$
A_{i}^{(r+1)}=X_{i}^{(r)} \#_{1-w_{i}} \mathfrak{G}_{n}\left(\hat{\omega}_{\neq i} ;\left(X_{k}^{(r)}\right)_{k \neq i}\right), \quad X_{i}^{(r)}=\sum_{k=1}^{n+1} w_{i k} A_{k}^{(r)} .
$$

By Theorem 3.1 (i) and Lemma 2.2,

$$
\begin{aligned}
d\left(X_{i}^{(r)}, X_{j}^{(r)}\right) & =d\left(\sum_{k=1}^{n+1} w_{i k} A_{k}^{(r)}, \sum_{l=1}^{n+1} w_{j l} A_{l}^{(r)}\right) \\
& =d\left(\sum_{k=1}^{n+1} w_{i k} A_{k}^{(r)}, \sum_{k=1}^{n+1} w_{i k}\left(\sum_{l=1}^{n+1} w_{j l} A_{l}^{(r)}\right)\right) \\
& \leq \max _{k} d\left(w_{i k} A_{k}^{(r)}, w_{i k} \sum_{l=1}^{n+1} w_{j l} A_{l}^{(r)}\right) \\
& =\max _{k} d\left(A_{k}^{(r)}, \sum_{l=1}^{n+1} w_{j l} A_{l}^{(r)}\right) \\
& =\max _{k} d\left(\sum_{l=1}^{n+1} w_{j l} A_{k}^{(r)}, \sum_{l=1}^{n+1} w_{j l} A_{l}^{(r)}\right) \\
& \leq \max _{k, l} d\left(w_{j l} A_{k}^{(r)}, w_{j l} A_{l}^{(r)}\right) \\
& =\Delta\left(A_{1}^{(r)}, \ldots, A_{n+1}^{(r)}\right)
\end{aligned}
$$


and

$$
\Delta\left(\left(X_{1}^{(r)}, \ldots, X_{n+1}^{(r)}\right)\right) \leq \Delta\left(A_{1}^{(r)}, \ldots, A_{n+1}^{(r)}\right)
$$

where $\Delta\left(A_{1}, \ldots, A_{n+1}\right)=\max _{1 \leq i, j \leq n+1}\left\{d\left(A_{i}, A_{j}\right)\right\}$, the diameter of $\left\{A_{i}\right\}_{i=1}^{n+1}$.

By Theorem 3.1 (3),

$$
\begin{aligned}
d\left(A_{i}^{(r+1)}, A_{j}^{(r+1)}\right)= & d\left(X_{i}^{(r)} \#_{1-w_{i}} \mathfrak{G}_{n}\left(\hat{\omega}_{\neq i} ;\left(X_{k}^{(r)}\right)_{k \neq i}\right), X_{j}^{(r)} \#_{1-w_{j}} \mathfrak{G}_{n}\left(\hat{\omega}_{\neq j} ;\left(X_{l}^{(r)}\right)_{l \neq j}\right)\right) \\
\leq & w_{i} w_{j} d\left(X_{i}^{(r)}, X_{j}^{(r)}\right) \\
& +w_{i}\left(1-w_{j}\right) d\left(X_{i}^{(r)}, \mathfrak{G}_{n}\left(\hat{\omega}_{\neq j} ;\left(X_{l}^{(r)}\right)_{l \neq j}\right)\right) \\
& +\left(1-w_{i}\right) w_{j} d\left(\mathfrak{G}_{n}\left(\hat{\omega}_{\neq i} ;\left(X_{k}^{(r)}\right)_{k \neq i}\right), X_{j}^{(r)}\right) \\
& +\left(1-w_{i}\right)\left(1-w_{j}\right) d\left(\mathfrak{G}_{n}\left(\hat{\omega}_{\neq i} ;\left(X_{k}^{(r)}\right)_{k \neq i}\right), \mathfrak{G}_{n}\left(\hat{\omega}_{\neq j} ;\left(X_{l}^{(r)}\right)_{l \neq j}\right) .\right.
\end{aligned}
$$

By Proposition 3.5, we have following inequalities

$$
\begin{aligned}
& d\left(\mathfrak{G}_{n}\left(\hat{\omega}_{\neq i} ;\left(X_{k}^{(r)}\right)_{k \neq i}\right), \mathfrak{G}_{n}\left(\hat{\omega}_{\neq j} ;\left(X_{l}^{(r)}\right)_{l \neq j}\right)\right) \\
& \leq \sum_{k \neq i} \sum_{l \neq j} \frac{w_{k}}{1-w_{i}} \frac{w_{l}}{1-w_{j}} d\left(X_{k}^{(r)}, X_{l}^{(r)}\right) \\
& =\sum_{k \neq i, l} \sum_{l \neq j} \frac{w_{k}}{1-w_{i}} \frac{w_{l}}{1-w_{j}} d\left(X_{k}^{(r)}, X_{l}^{(r)}\right)+\sum_{l \neq i, j} \frac{w_{l}}{1-w_{i}} \frac{w_{l}}{1-w_{j}} d\left(X_{l}^{(r)}, X_{l}^{(r)}\right) \\
& \leq \sum_{k \neq i, l} \sum_{l \neq j} \frac{w_{k}}{1-w_{i}} \frac{w_{l}}{1-w_{j}} \Delta\left(X_{1}^{(r)}, \ldots, X_{n+1}^{(r)}\right) \\
& =\left(1-\sum_{l \neq i, j} \frac{w_{l}}{1-w_{i}} \frac{w_{l}}{1-w_{j}}\right) \Delta\left(X_{1}^{(r)}, \ldots, X_{n+1}^{(r)}\right) \\
& d\left(X_{i}^{(r)}, \mathfrak{G}_{n}\left(\hat{\omega}_{\neq j} ;\left(X_{l}^{(r)}\right)_{l \neq j}\right)\right) \leq \sum_{l \neq j} \frac{w_{l}}{1-w_{j}} d\left(X_{i}^{(r)}, X_{l}^{(r)}\right) \\
& =\sum_{l \neq i, j} \frac{w_{l}}{1-w_{j}} d\left(X_{i}^{(r)}, X_{l}^{(r)}\right)+\frac{w_{i}}{1-w_{j}} d\left(X_{i}^{(r)}, X_{i}^{(r)}\right) \\
& \leq \sum_{l \neq i, j} \frac{w_{l}}{1-w_{j}} \Delta\left(X_{1}^{(r)}, \ldots, X_{n+1}^{(r)}\right) \\
& =\left(1-\frac{w_{i}}{1-w_{j}}\right) \Delta\left(X_{1}^{(r)}, \ldots, X_{n+1}^{(r)}\right)
\end{aligned}
$$

and

$$
d\left(\mathfrak{G}_{n}\left(\hat{\omega}_{\neq i} ;\left(X_{k}^{(r)}\right)_{k \neq i}\right), X_{j}^{(r)}\right) \leq\left(1-\frac{w_{j}}{1-w_{i}}\right) \Delta\left(X_{1}^{(r)}, \ldots, X_{n+1}^{(r)}\right)
$$


Therefore

$$
\begin{aligned}
& d\left(A_{i}^{(r+1)}, A_{j}^{(r+1)}\right) \\
& \leq\left(w_{i} w_{j}+w_{i}\left(1-w_{j}\right)\left(1-\frac{w_{i}}{1-w_{j}}\right)+\left(1-w_{i}\right) w_{j}\left(1-\frac{w_{j}}{1-w_{i}}\right)\right. \\
& \left.\quad+\left(1-w_{i}\right)\left(1-w_{j}\right)\left(1-\sum_{l \neq i, j} \frac{w_{l}}{1-w_{i}} \frac{w_{l}}{1-w_{j}}\right)\right) \Delta\left(X_{1}^{(r)}, \ldots, X_{n+1}^{(r)}\right) \\
& =\left(1-\sum_{k=1}^{n+1} w_{k}^{2}\right) \Delta\left(X_{1}^{(r)}, \ldots, X_{n+1}^{(r)}\right) \\
& \leq\left(1-\sum_{k=1}^{n+1} w_{k}^{2}\right) \Delta\left(A_{1}^{(r)}, \ldots, A_{n+1}^{(r)}\right) .
\end{aligned}
$$

the last inequality follows by (4.9). Inductively, we have

$$
\begin{aligned}
d\left(A_{i}^{(r)}, A_{j}^{(r)}\right) & \leq\left(1-\sum_{k=1}^{n+1} w_{k}^{2}\right) \Delta\left(A_{1}^{(r-1)}, \ldots, A_{n+1}^{(r-1)}\right) \\
& \leq \cdots \leq\left(1-\sum_{k=1}^{n+1} w_{k}^{2}\right)^{r} \Delta\left(A_{1}^{(0)}, \ldots, A_{n+1}^{(0)}\right) .
\end{aligned}
$$

By Lemma 2.2,

$$
\begin{aligned}
d\left(A_{i}^{(r)}, X_{j}^{(r)}\right) & =d\left(A_{i}^{(r)}, \sum_{k \neq j} w_{j k} A_{k}^{(r)}\right)=d\left(\sum_{k \neq j} w_{j k} A_{i}^{(r)}, \sum_{k \neq j} w_{j k} A_{k}^{(r)}\right) \\
& \leq \max _{k \neq j}\left\{d\left(A_{i}^{(r)}, A_{k}^{(r)}\right)\right\} \stackrel{(4.7)}{\leq}\left(1-\sum_{k=1}^{n+1} w_{k}^{2}\right)^{r} \Delta(A)
\end{aligned}
$$

and therefore

$$
\begin{aligned}
d\left(A_{i}^{(r)}, A_{i}^{(r+1)}\right) & =d\left(A_{i}^{(r)}, X_{i}^{(r)} \#_{1-w_{i}} \mathfrak{G}_{n}\left(\hat{w}_{\neq i} ;\left(X_{k}^{(r)}\right)_{k \neq i}\right)\right) \\
& \leq w_{i} d\left(A_{i}^{(r)}, X_{i}^{(r)}\right)+\left(1-w_{i}\right) d\left(A_{i}^{(r)}, g\left(\left(X_{k}^{(r)}\right)_{k \neq i}\right)\right) \\
& \leq w_{i} d\left(A_{i}^{(r)}, X_{i}^{(r)}\right)+\sum_{k \neq i} w_{k} d\left(A_{i}^{(r)}, X_{k}^{(r)}\right) \\
& \leq w_{i}\left(1-\sum_{k=1}^{n+1} w_{k}^{2}\right)^{r} \Delta(A)+\sum_{k \neq i} w_{k}\left(1-\sum_{k=1}^{n+1} w_{k}^{2}\right)^{r} \Delta(A) \\
& =\left(1-\sum_{k=1}^{n+1} w_{k}^{2}\right)^{r} \Delta(A) .
\end{aligned}
$$


This together with (4.7) shows that the sequences $A_{i}^{(r)}, 1 \leq i \leq n+1$, have a common limit.

The proof for $\mu_{\omega}$ is similar to that of $\delta_{\omega}$ by using the invariancy of the Thompson metric and the weighted geometric mean under the inversion (Lemma 3.2).

Definition 4.2. We denote $\mathfrak{C}(\omega ; A)\left(\operatorname{resp} . \mathfrak{C}^{*}(\omega ; A)\right)$ by the common limit of the iteration $\delta_{\omega}=\gamma_{\omega} \circ \beta_{\omega}\left(\right.$ resp. $\left.\mu_{\omega}\right)$ at $A$.

Remark 4.3. We consider the iterative mean algorithm $(\beta \circ \gamma)(A)$. From $(\beta \circ$ $\gamma)^{r+1}=\beta \circ(\gamma \circ \beta)^{r} \circ \gamma=\beta \circ \delta^{r} \circ \gamma$, we have $(\beta \circ \gamma)^{r+1}(A)=\beta\left(\delta^{r}(\gamma(A))\right)$. Passing to the limit as $r \rightarrow \infty$ yields

$$
\begin{aligned}
\lim _{r \rightarrow \infty}(\beta \circ \gamma)^{r+1}(A) & =\beta\left(\lim _{r \rightarrow \infty} \delta^{r}(\gamma(A))\right)=\beta(\mathfrak{C}(\omega ; \gamma(A)), \ldots, \mathfrak{C}(\omega ; \gamma(A))) \\
& =(\mathfrak{C}(\omega ; \gamma(A)), \ldots, \mathfrak{C}(\omega ; \gamma(A)))
\end{aligned}
$$

where the last equality follows from the fact that $\beta(A, A, \ldots, A)=(A, A, \ldots, A)$ for all $A>0$.

One may have interest in finding some properties of $\mathfrak{C}(\omega ; A)$ and $\mathfrak{C}^{*}(\omega ; A)$. The following results actually show that most of common properties of the arithmetic mean and the weighted geometric mean $\mathfrak{G}_{n}$ are preserved by $\mathfrak{C}$.

Theorem 4.4. The map $\mathfrak{C}: \Delta_{n+1} \times \Omega^{n+1} \rightarrow \Omega$ satisfies the following properties; for $A=\left(A_{1}, A_{2}, \ldots, A_{n+1}\right), B=\left(B_{1}, B_{2}, \ldots, B_{n+1}\right) \in \Omega^{n+1}$, a permutation $\sigma$ on $n+1$-letters, an invertible matrix $M$, and for $0 \leq \lambda \leq 1$,

(C1) (Idempotency) $\mathfrak{C}(\omega ; A, A, \ldots, A)=A$;

(C2) (Homogeneity) for any $s>0, \mathfrak{C}(\omega ; s A)=s \mathfrak{C}(\omega ; A)$;

(C3) (Permutation symmetry) $=\mathfrak{C}\left(\omega_{\sigma} ; A_{\sigma(1)}, \ldots, A_{\sigma(n+1)}\right)$;

(C4) (Monotonicity) If $B_{i} \leq A_{i}$ for all $i$, then $\mathfrak{C}(\omega ; B) \leq \mathfrak{C}(\omega ; A)$;

(C5) (Continuity) $\mathfrak{C}(\omega ; \cdot)$ is continuous;

(C6) (Congruence Invariancy) $\mathfrak{C}\left(\omega ; M A_{1} M^{*}, \ldots, M A_{n} M^{*}\right)=M \mathfrak{C}(\omega ; A) M^{*}$;

(C7) (Joint Concavity) $\mathfrak{C}(\omega ; \lambda A+(1-\lambda) B) \geq \lambda \mathfrak{C}(\omega ; A)+(1-\lambda) \mathfrak{C}(\omega ; B)$;

(C8) (Duality) $\left(\mathfrak{C}\left(\omega ; A^{-1}\right)\right)^{-1}=\mathfrak{C}^{*}(\omega ; A)$;

For regular stochastic matrices

$$
R_{1}=\left[\begin{array}{ccccc}
w_{1} & w_{2} & w_{3} & \cdots & w_{n+1} \\
w_{1} & w_{2} & w_{3} & \cdots & w_{n+1} \\
\vdots & \vdots & \vdots & \ddots & \vdots \\
w_{1} & w_{2} & w_{3} & \cdots & w_{n+1}
\end{array}\right]
$$




$$
R_{2}=\left[\begin{array}{ccccc}
0 & \frac{w_{2}}{1-w_{1}} & \frac{w_{3}}{1-w_{1}} & \cdots & \frac{w_{n+1}}{1-w_{1}} \\
\frac{w_{1}}{1-w_{2}} & 0 & \frac{w_{3}}{1-w_{2}} & \cdots & \frac{w_{n+1}}{1-w_{2}} \\
\vdots & \vdots & \vdots & \ddots & \vdots \\
\frac{w_{1}}{1-w_{n+1}} & \frac{w_{2}}{1-w_{n+1}} & \frac{w_{3}}{1-w_{n+1}} & \cdots & 0
\end{array}\right] .
$$

and an eigenvector $z=\left(z_{1}, \ldots, z_{n+1}\right)^{T}$ of the regular stochastic matrix $\left(R_{1} R_{2}\right)^{T}$ corresponding to the eigenvalue 1, we have

(C9) (Determinantal inequality) $\operatorname{det} \mathfrak{C}(\omega ; A) \leq \prod_{i=1}^{n+1}\left(\operatorname{det} A_{i}\right)^{z_{i}}$;

(C10) (ACH mean inequalities)

$$
\left(\sum_{k=1}^{n+1} z_{k} A_{k}^{-1}\right)^{-1} \leq \mathfrak{C}^{*}(\omega ; \mathbb{A}) \leq \mathfrak{C}(\omega ; \mathbb{A}) \leq \sum_{k=1}^{n+1} z_{k} A_{k}
$$

Proof. Let $A=\left(A_{1}, \ldots, A_{n+1}\right), B=\left(B_{1}, \ldots, B_{n+1}\right) \in \Omega^{n+1}$. Set $\gamma=\gamma_{\omega}, \delta=\delta_{\omega}$ and

$$
\begin{aligned}
\delta_{\omega}^{r}(A)=\left(A_{1}^{(r)}, \ldots, A_{n+1}^{(r)}\right), & \delta_{\omega}^{r}(B)=\left(B_{1}^{(r)}, \ldots, B_{n+1}^{(r)}\right) \\
\left(\beta_{\omega} \circ \delta_{\omega}^{r}\right)(A)=\left(X_{1}^{(r)}, \ldots, X_{n+1}^{(r)}\right), & \left(\beta_{\omega} \circ \delta_{\omega}^{r}\right)(B)=\left(Y_{1}^{(r)}, \ldots, Y_{n+1}^{(r)}\right) .
\end{aligned}
$$

We consider the partial order on $\Omega^{n+1} ; B \leq A$ if and only if $B_{i} \leq A_{i}, \quad i=$ $1, \ldots, n+1$. One may see that $\beta_{\omega}$ and $\gamma_{\omega}$ are monotone functions by the monotonicity of $\mathfrak{G}_{n}$ and two-variable weighted geometric means ((P4) and Lemma 3.2). In particular, $\delta_{\omega}=\gamma_{\omega} \circ \beta_{\omega}$ is monotone.

(C1) It follows from $\beta_{\omega}(A, \ldots, A)=\gamma_{\omega}(A, \ldots, A)=(A, \ldots, A)$.

(C2) It follows from $\beta_{\omega}(s A)=s \beta_{\omega}(A)$ and $\gamma_{\omega}(s A)=s \gamma_{\omega}(A)$.

(C3) Let $\sigma$ be a permutation on $(n+1)$-letters. Put $\omega_{\sigma}=\left(w_{\sigma(1)}, w_{\sigma(2)}, \ldots, w_{\sigma(n+1)}\right)$. We consider the sequences $\left\{B_{i}^{(r)}\right\}_{r=0}^{\infty}$ which determine the $\omega_{\sigma}$-weighted Carlson mean $\mathfrak{G}_{n}\left(\omega_{\sigma} ; A_{\sigma}\right)$. By definition, $B_{i}^{(0)}=B_{i}=A_{\sigma(i)}=A_{\sigma(i)}^{(0)}$ for all $i$. Suppose that $B_{i}^{(r)}=A_{\sigma(i)}^{(r)}$ for all $i$. Then $Y_{i}^{(r)}=\sum_{k \neq i} w_{\sigma(k)} B_{k}^{(r)}=\sum_{k \neq i} w_{\sigma(k)} A_{\sigma(k)}^{(r)}=$ $\sum_{k \neq \sigma(i)} w_{k} A_{k}^{(r)}=X_{\sigma(i)}^{(r)}$ for all $i$. By the permutation invariancy of $\mathfrak{G}_{n}$,

$$
\begin{aligned}
B_{i}^{(r+1)} & =Y_{i}^{(r)} \#_{1-w_{\sigma(i)}} \mathfrak{G}_{n}\left(\widehat{\left(\omega_{\sigma}\right)_{\neq i}} ;\left(Y_{k}^{(r)}\right)_{k \neq i}\right) \\
& =X_{\sigma(i)}^{(r)} \#_{1-w_{\sigma(i)}} \mathfrak{G}_{n}\left(\widehat{\left(\omega_{\sigma}\right)_{\neq i}} ; X_{\sigma(1)}^{(r)}, \ldots, X_{\sigma(i-1)}^{(r)}, X_{\sigma(i+1)}^{(r)}, \ldots X_{\sigma(n+1)}^{(r)}\right) \\
& =X_{\sigma(i)}^{(r)} \#_{1-w_{\sigma(i)}} \mathfrak{G}_{n}\left(\hat{\omega}_{\neq \sigma(i)} ; X_{1}^{(r)}, \ldots, X_{\sigma(i)-1}^{(r)}, X_{\sigma(i)+1}^{(r)}, \ldots X_{n+1}^{(r)}\right) \\
& =X_{\sigma(i)}^{(r)} \#_{1-w_{\sigma(i)}} \mathfrak{G}_{n}\left(\hat{\omega}_{\neq \sigma(i)} ;\left(X_{k}^{(r)}\right)_{k \neq \sigma(i)}\right)=A_{\sigma(i)}^{(r+1)} .
\end{aligned}
$$

By definition of $\mathfrak{C}(\omega ; A)$ and $\mathfrak{C}\left(\omega_{\sigma} ; A_{\sigma}\right)$, we have

$$
\mathfrak{C}\left(\omega ; A_{\sigma(1)}, A_{\sigma(2)}, \ldots, A_{\sigma(n+1)}\right)=\lim _{r \rightarrow \infty} B_{i}^{(r)}=\lim _{r \rightarrow \infty} A_{\sigma(i)}^{(r)}=\mathfrak{C}(\omega ; A) .
$$


(C4) Let $B_{i}>0$ with $B_{i} \leq A_{i}$ for all $i=1, \ldots, n+1$. Then $\beta_{\omega}(B) \leq \beta_{\omega}(A)$ and $\gamma_{\omega}(B) \leq \gamma_{\omega}(A)$ and hence $\delta_{\omega}(B)=\left(\gamma_{\omega} \circ \beta_{\omega}\right)(B)=\gamma_{\omega}\left(\beta_{\omega}(B)\right) \leq \gamma_{\omega}\left(\beta_{\omega}(A)\right)=$ $\left(\gamma_{\omega} \circ \beta_{\omega}\right)(A)=\delta_{\omega}(A)$. Since $\leq$ is closed in $\Omega$ and $\delta_{\omega}^{r}(B) \leq \delta_{\omega}^{r}(A)$, we conclude that $\mathfrak{C}(\omega ; B) \leq \mathfrak{C}(\omega ; A)$.

(C5) By Lemma 2.2,

$$
\begin{aligned}
d\left(X_{i}^{(r)}, Y_{i}^{(r)}\right) & =d\left(\sum_{k \neq i} \frac{w_{k}}{1-w_{i}} A_{k}^{(r)}, \sum_{k \neq i} \frac{w_{k}}{1-w_{i}} B_{k}^{(r)}\right) \\
& \leq \max _{k \neq i} d\left(\frac{w_{k}}{1-w_{i}} A_{k}^{(r)}, \frac{w_{k}}{1-w_{i}} B_{k}^{(r)}\right)=\max _{k \neq i} d\left(A_{k}^{(r)}, B_{k}^{(r)}\right) \\
& \leq \max _{j} d\left(A_{j}^{(r)}, B_{j}^{(r)}\right)
\end{aligned}
$$

for all $1 \leq i \leq n+1$. By (3.1) and by $(P 11)$,

$$
\begin{aligned}
d\left(A_{i}^{(r+1)}\right. & \left., B_{i}^{(r+1)}\right) \\
& =d\left(X_{i}^{(r)} \#_{1-w_{i}} \mathfrak{G}_{n}\left(\hat{\omega}_{\neq i} ;\left(X_{k}^{(r)}\right)_{k \neq i}\right), Y_{i}^{(r)} \#_{1-w_{i}} \mathfrak{G}_{n}\left(\hat{\omega}_{\neq i} ;\left(Y_{k}^{(r)}\right)_{k \neq i}\right)\right) \\
& \leq w_{i} d\left(X_{i}^{(r)}, Y_{i}^{(r)}\right)+\left(1-w_{i}\right) d\left(\mathfrak{G}_{n}\left(\hat{\omega}_{\neq i} ;\left(X_{k}^{(r)}\right)_{k \neq i}\right), \mathfrak{G}_{n}\left(\hat{\omega}_{\neq i} ;\left(Y_{k}^{(r)}\right)_{k \neq i}\right)\right) \\
& \leq w_{i} d\left(X_{i}^{(r)}, Y_{i}^{(r)}\right)+\left(1-w_{i}\right) \sum_{k \neq i} \frac{w_{k}}{1-w_{i}} d\left(X_{k}^{(r)}, Y_{k}^{(r)}\right) \\
& =\sum_{k=1}^{n+1} w_{k} d\left(X_{k}^{(r)}, Y_{k}^{(r)}\right) \\
& \leq \max _{j} d\left(A_{j}^{(r)}, B_{j}^{(r)}\right)
\end{aligned}
$$

and therefore

$$
d\left(A_{i}^{(r)}, B_{i}^{(r)}\right) \leq \max _{j} d\left(A_{j}^{(r-1)}, B_{j}^{(r-1)}\right) \leq \cdots \leq \max _{j} d\left(A_{j}^{(0)}, B_{j}^{(0)}\right)=\max _{j} d\left(A_{j}, B_{j}\right)
$$

Passing to the limit as $r \rightarrow \infty$ yields $d(\mathfrak{C}(\omega ; A), \mathfrak{C}(\omega ; B)) \leq \max _{j} d\left(A_{j}, B_{j}\right)$.

(C6) It follows from $\beta_{\omega}\left(M A M^{*}\right)=M \beta_{\omega}(A) M^{*}$ and $\gamma_{\omega}\left(M A M^{*}\right)=M \gamma_{\omega}(A) M^{*}$ where $M A M^{*}=\left(M A_{1} M^{*}, \ldots, M A_{n+1} M^{*}\right)$.

(C7) Let $\left\{C_{i}^{(r)}\right\}_{r=0}^{\infty}$ (resp. $\left\{Z_{i}^{(r)}\right\}_{r=0}^{\infty}$ ) be defined in the same fashion as $\left\{A_{i}^{(r)}\right\}_{r=0}^{\infty}$ (resp. $\left\{X_{i}^{(r)}\right\}_{r=0}^{\infty}$ ), but starting from $C_{i}=(1-\lambda) A_{i}+\lambda B_{i}$.

We shall show that $C_{i}^{(r)} \geq(1-\lambda) A_{i}^{(r)}+\lambda B_{i}^{(r)}$ for all $r$ and $i$. From $A_{i}^{(0)}=A_{i}, B_{i}^{(0)}=$ $B_{i}, C_{i}^{(0)}=C_{i}$, it holds true for $r=0$. Suppose that $C_{i}^{(r)} \geq(1-\lambda) A_{i}^{(r)}+\lambda B_{i}^{(r)}$ for 
all $i=1,2, \ldots, n+1$. Then

$$
\begin{aligned}
Z_{i}^{(r)} & =\sum_{k \neq i} w_{k} C_{k}^{(r)} \\
& \geq \sum_{k \neq i} w_{k}\left((1-\lambda) A_{k}^{(r)}+\lambda B_{k}^{(r)}\right) \\
& =(1-\lambda) \sum_{k \neq i} w_{k} A_{k}^{(r)}+\lambda \sum_{k \neq i} w_{k} B_{k}^{(r)} \\
& =(1-\lambda) X_{i}^{(r)}+\lambda Y_{i}^{(r)}
\end{aligned}
$$

for all $i=1,2, \ldots, n+1$. By the monotonicity and concavity of $\mathfrak{G}_{n}((\mathrm{P} 4),(\mathrm{P} 7))$ and two-variable weighted geometric means (Lemma 3.2),

$$
\begin{aligned}
C_{i}^{(r+1)}= & Z_{i}^{(r)} \#_{1-w_{i}} \mathfrak{G}_{n}\left(\hat{\omega}_{\neq i} ;\left(Z_{k}^{(r)}\right)_{k \neq i}\right) \\
\geq & \left((1-\lambda) X_{i}^{(r)}+\lambda Y_{i}^{(r)}\right) \#_{1-w_{i}} \mathfrak{G}_{n}\left(\hat{\omega}_{\neq i} ;\left((1-\lambda) X_{k}^{(r)}+\lambda Y_{k}^{(r)}\right)_{k \neq i}\right) \\
\geq & \left((1-\lambda) X_{i}^{(r)}+\lambda Y_{i}^{(r)}\right) \#_{1-w_{i}} \\
& \quad\left((1-\lambda) \mathfrak{G}_{n}\left(\hat{\omega}_{\neq i} ;\left(X_{k}^{(r)}\right)_{k \neq i}\right)+\lambda \mathfrak{G}_{n}\left(\hat{\omega}_{\neq i} ;\left(Y_{k}^{(r)}\right)_{k \neq i}\right)\right) \\
\geq & (1-\lambda)\left(X_{i}^{(r)} \#_{1-w_{i}} \mathfrak{G}_{n}\left(\hat{\omega}_{\neq i} ;\left(X_{k}^{(r)}\right)_{k \neq i}\right)\right) \\
& \quad+\lambda\left(Y_{i}^{(r)} \#_{1-w_{1}} \mathfrak{G}_{n}\left(\hat{\omega}_{\neq i} ;\left(Y_{k}^{(r)}\right)_{k \neq i}\right)\right) \\
= & (1-\lambda) A_{i}^{(r+1)}+\lambda B_{i}^{(r+1)} .
\end{aligned}
$$

Passing to the limit as $r \rightarrow \infty$ yields

$\mathfrak{C}(\omega ;(1-\lambda) A+\lambda B)=\lim _{r \rightarrow \infty} C_{i}^{(r)} \geq \lim _{r \rightarrow \infty}\left((1-\lambda) A_{i}^{(r+1)}+\lambda B_{i}^{(r+1)}\right)=(1-\lambda) \mathfrak{C}(\omega ; A)+\lambda \mathfrak{C}(\omega ; B)$.

(C8) It follows from $\beta_{\omega}\left(A^{-1}\right)^{-1}=\beta_{\omega}^{*}(A)$ and $\gamma_{\omega}\left(A^{-1}\right)^{-1}=\gamma_{\omega}(A)$ (Lemma 3.2

(v)) where $A^{-1}=\left(A_{1}^{-1}, \ldots, A_{n+1}^{-1}\right)$.

(C9) By the determinant identity of $\mathfrak{G}_{n}$ (P9) and two-variable weighted geometric means (Lemma 3.2),

$$
\begin{aligned}
\operatorname{det} A_{i}^{(r+1)} & =\operatorname{det}\left(X_{i}^{(r)} \#_{1-w_{i}} \mathfrak{G}_{n}\left(\hat{\omega}_{\neq i} ;\left(X_{k}^{(r)}\right)_{k \neq i}\right)\right) \\
& =\left(\operatorname{det} X_{i}^{(r)}\right)^{w_{i}}\left(\operatorname{det} \mathfrak{G}_{n}\left(\hat{\omega}_{\neq i} ;\left(X_{k}^{(r)}\right)_{k \neq i}\right)\right)^{1-w_{i}} \\
& =\left(\operatorname{det} X_{i}^{(r)}\right)^{w_{i}}\left(\prod_{k \neq i}\left(\operatorname{det} X_{k}^{(r)}\right)^{\frac{w_{k}}{1-w_{i}}}\right)^{1-w_{i}} \\
& =\prod_{k=1}^{n+1}\left(\operatorname{det} X_{k}^{(r)}\right)^{w_{k}}
\end{aligned}
$$


and

$$
\operatorname{det} X_{i}^{(r)}=\operatorname{det}\left(\sum_{k \neq i} \frac{w_{k}}{1-w_{i}} A_{i}^{(r)}\right) \geq \prod_{k \neq i} \operatorname{det} A_{i}^{\frac{w_{k}}{11-w_{i}}}
$$

where the inequality follows by Corollary 7.6.9 of [9] for $n=2$ and by an appropriate symmetrization method for $n>2$.

Setting $\mathbf{a}^{(r)}=\left(a_{1}^{(r)}, \ldots, a_{n+1}^{(r)}\right)$ and $\mathbf{x}^{(r)}=\left(x_{1}^{(r)}, \ldots, x_{n+1}^{(r)}\right)$ where $a_{i}^{(r)}=\log \operatorname{det} A_{i}^{(r)}$ and $x_{i}^{(r)}=\log \operatorname{det} X_{i}^{(r)}$, respectively. Then we have

$$
a_{i}^{(r+1)}=\sum_{k=1}^{n+1} w_{k} x_{k}^{(r)} \quad \text { and } \quad x_{i}^{(r)} \geq \sum_{k \neq i} \frac{w_{k}}{1-w_{i}} a_{k}
$$

and these relations can be rewritten by using the regular stochastic matrices $R_{1}$ and $R_{2}$ as

$$
\mathbf{a}^{(r+1)}=R_{1} \mathbf{x}^{(r)} \quad \text { and } \quad \mathbf{x}^{(r)} \geq R_{2} \mathbf{a}^{(r)} .
$$

Since every row of $R_{1}$ is nonnegative probability vector,

$$
\mathbf{a}^{(r+1)} \geq R_{1} R_{2} \mathbf{a}^{(r)}
$$

and hence, inductively, we have

$$
\mathbf{a}^{(r)} \geq\left(R_{1} R_{2}\right) \mathbf{a}^{(r-1)} \geq \cdots \geq\left(R_{1} R_{2}\right)^{r} \mathbf{a}^{(0)} .
$$

From the facts that every component of $\mathbf{a}^{(r)}$ converges to $\log \operatorname{det} \mathfrak{C}(\omega ; A)$ and the sequence $\left\{\left(R_{1} R_{2}\right)^{r}\right\}$ converges to a matrix $S$ whose rows are the fixed row $z=$ $\left(z_{1}, \ldots, z_{n+1}\right)$, we obtain

$$
\operatorname{det} \mathfrak{C}(\omega ; A) \geq \prod_{i=1}^{n+1}\left(\operatorname{det} A_{i}\right)^{z_{i}}
$$

(C10) From the arithmetic-harmonic mean inequality, we have $\beta_{\omega}^{*}(\mathbb{A}) \leq \beta_{\omega}(\mathbb{A})$ and hence by the monotonicity of $\gamma_{\omega}, \mu_{\omega}(A)=\gamma_{\omega}\left(\beta_{\omega}^{*}(A)\right) \leq \gamma_{\omega}\left(\beta_{\omega}(A)\right)=\delta_{\omega}(A)$. By monotonicity of $\delta_{\omega}$ and by induction, $\mu_{\omega}^{r}(A) \leq \delta_{\omega}^{r}(A)$ for all $r$ and therefore $\mathfrak{C}^{*}(\omega ; \mathbb{A}) \leq \mathfrak{C}(\omega ; \mathbb{A})$.

We consider a self-map on $\Omega^{n+1}$ defined by $\mu(\mathbb{A})=\left(\sum_{k=1}^{n+1} w_{k} A_{k}, \ldots, \sum_{k=1}^{n+1} w_{k} A_{k}\right)$ It is not difficult to see that $\lim _{r \rightarrow \infty}(\mu \circ \beta)^{r}(\mathbb{A})=(X, \ldots, X)$ and $X=\sum_{i=1}^{n+1} z_{i} A_{i}$ by using its linear representation

$$
A \mapsto\left(R_{1} R_{2}\right) A
$$

and the corresponding matrix $\left(R_{1} R_{2}\right)$ is transpose of a regular stochastic matrix with an eigenvector $z=\left(z_{1}, z_{2}, \ldots, z_{n}\right)$ of the eigenvalue 1 . 
We will show by induction that $\delta^{r}(\mathbb{A}) \leq(\mu \circ \beta)^{r}(\mathbb{A})$ for all positive integers $r$, which implies that $\mathfrak{C}(\omega ; \mathbb{A}) \leq X=\sum_{i=1}^{n+1} z_{i} A_{i}$ by passing to the limit as $r \rightarrow \infty$. By the arithmetic-geometric mean inequality (Lemma 3.2) and (P10),

$$
\begin{aligned}
A_{i} \#_{1-w_{i}} \mathfrak{G}_{n}\left(\hat{\omega}_{\neq i} ;\left(A_{k}\right)_{k \neq i}\right) & \leq w_{i} A_{i}+\left(1-w_{i}\right) \mathfrak{G}_{n}\left(\hat{\omega}_{\neq i} ;\left(A_{k}\right)_{k \neq i}\right) \\
& \leq w_{i} A_{i}+\left(1-w_{i}\right) \sum_{k \neq i} \frac{w_{k}}{1-w_{i}} A_{k} \\
& =\sum_{k=1}^{n+1} w_{k} A_{k}
\end{aligned}
$$

for all $i$ and therefore $\gamma(\mathbb{A}) \leq \mu(\mathbb{A})$. Replacing $\mathbb{A}$ to $\beta(\mathbb{A})$ yields $\delta(\mathbb{A})=(\gamma \circ \beta)(A)=$ $\gamma(\beta(A)) \leq(\mu \circ \beta)(\mathbb{A})$. Suppose that $\delta^{r}(\mathbb{A}) \leq(\mu \circ \beta)^{r}(\mathbb{A})$. Then by the monotonicity of $\delta, \delta^{r+1}(\mathbb{A})=\delta\left(\delta^{r}(\mathbb{A})\right) \leq \delta\left((\mu \circ \beta)^{r}(\mathbb{A})\right) \leq(\mu \circ \beta)\left((\mu \circ \beta)^{r}(\mathbb{A})\right)=(\mu \circ \beta)^{r+1}(\mathbb{A})$.

The inequality $\left(\sum_{k=1}^{n+1} z_{k} A_{k}^{-1}\right)^{-1} \leq \mathfrak{C}^{*}(\omega ; \mathbb{A})$ follows by the preceding one and the duality (C8).

\section{References}

[1] T. Ando, C.K. Li and R. Mathias, Geometric means, Linear Algebra Appl., 385(2004), 305-334.

[2] R. Bhatia, On the exponential metric increasing property, Linear Algebra Appl. 375(2003), 211-220.

[3] R. Bhatia, Positive definite matrices, Princeton Series in Applied Mathematics, Princeton University Press, Princeton, NJ, 2007.

[4] D. Bini, B. Meini and F. Poloni, An effective matrix geometric mean satisfying the Ando-Li-Mathias properties, Math. Comp., 79(2010), 437-452.

[5] C. W. Borchardt, Sur deux algorithmes analogues á celui de la moyenne arithéticogéométrique de deux éléments, In memoriiam Dominici Chelini, Collectanea Mathematica [etc], L. Cremona, ed., U. Hoepli, Milan, 1881, 455-462.

[6] B. C. Carlson, Hidden symmetries of special functions, SIAM Review, 12(1970), 332346 .

[7] B. C. Carlson, Algorithms involving arithmetic and geometric means, Amer. Math. Monthly, 78(1971), 496-505.

[8] G. Corach, H. Porta, and L. Recht, Convexity of the geodesic distance on spaces of positive operators, Illinois J. Math. 38(1994), 87-94.

[9] R. Horn and C. Johnson, Matrix analysis, Cambridge University Press, Cambridge, 1985.

[10] S. Lang, Fundamentals of differential geometry, Graduate Texts in Math., Springer, Heidelberg, 1999.

[11] J. D. Lawson H. Lee and Y. Lim, Weighted geometric means, Forum Mathematicum, 24(2012), 1067-1090. 
[12] J. D. Lawson and Y. Lim, The geometric mean, matrices, metrics, and more, Amer. Math. Monthly 108(2001), 797-812.

[13] H. Lee and Y. Lim, Carlson's iterative algorithm of positive definite matrices, Linear Algebra Appl., accepted

[14] H. Lee and Y. Lim, Invariant metrics, contractions and nonlinear matrix equations, Nonlinearity 21(2008), 857-878. 